\title{
Nanoleakage and Durability of Resin/Dentin Bonds
}

\author{
Masanori Hashimoto $^{1} \cdot$ Satoshi Yamaguchi $^{1} \cdot$ Satoshi Imazato $^{1}$
}

Published online: 25 August 2015

(C) Springer International Publishing AG 2015

\begin{abstract}
Micromorphological analysis is a useful method for the evaluation of resin-dentin bonds. Transmission electron microscopy (TEM) can also be used to analyze the structure of resin-dentin bonds because of its high resolution. There are several reasons for the degradation of resin-dentin bonds such as the activation of endogenous dentin matrix metalloproteinases and the hydrolysis of methacrylate resins. Nanoleakage expression is an effective method for the identification of these degradation pathways and uses silver nitrate as a nanoleakage tracer. However, the resulting TEM images only provide two-dimensional information and the corresponding three-dimensional structure can only be realized based on the consideration of other experimental findings. The evaluation of nanoleakage structures in this way should provide a better understanding of the bond degradation pathways. This review provides an overview of recent developments towards the study of nanoleakage structures, with particular emphasis on their shape and bond degradation processes.
\end{abstract}

Keywords Resin-dentin bonds · Nanoleakage $\cdot$ Water tree . Silver nitrate $\cdot$ Degradation $\cdot$ Durability

This article is part of the Topical Collection on Dental Restorative Materials

Masanori Hashimoto

masanori-h@mue.biglobe.ne.jp

Satoshi Yamaguchi

yamagu@dent.osaka-u.ac.jp

Satoshi Imazato

Imazato@dent.osaka-u.ac.jp

1 Department of Biomaterials Science, Graduate School of Dentistry, Osaka University, 1-8 Yamadaoka, Suita, Osaka 565-0871, Japan

\section{Introduction}

In general, the degradation of resin-dentin bonds can be categorized into three major groups, including (1) the hydrolytic degradation of the collagen matrix, (2) the hydrolytic degradation of the bonding resin within the hybrid layer, and (3) the hydrolytic degradation of the resin [1-4]. In this review, we have focused specifically on the nanoleakage or water tree extension of the resin-dentin bonds. Although the perfect encapsulation of the collagen matrix by the resin monomers is necessary for the formation of an ideal hybrid layer, the imperfect penetration of the resin into the collage web is inevitable for total-etch adhesives and, as well, to a lesser extent, self-etching adhesive systems [5, 6]. The use of an adhesive resin can yield permeable and unstable bonds that are susceptible to water sorption, which can lead to the hydrolytic degradation of the bonds [7-10]. This tendency towards degradation has become increasingly pronounced in some recently developed adhesive systems such as the one-bottle resin systems because of their hydrophilicity [8-10]. The hydrophilic nature of these systems is caused by their polar ingredients (e.g., acetone, ethanol, and water), which are necessary for the flowability of the adhesives or the ionization of the acidic monomers $[10,11]$. The hydrolysis of the bonds generally correlates well with the morphology and extent of the nanoleakage $[12,13]$. Water molecules can penetrate into the adhesive interface via nanoleakage, and the subsequent hydrolytic degradation of the bonds leads to the widening of the nanoleakage pathway [14-16]. This aging process can also increase the degradation of the resin by other hydrolytic pathways as well as enhance the hydrolytic activity of host-derived proteases for the collagen fibrils in the bonds $[17 \bullet, 18 \bullet \bullet, 19$, 20]. Nanoleakage and water tree structures can be traced by ammoniacal silver nitrate and observed by scanning electron microscopy (SEM) or transmission electron microscopy 
(TEM) $[21,22]$. However, the morphological analysis of nanoleakage or water tree by SEM and TEM is generally conducted as a two-dimensional process and additional steps are required to provide a three-dimensional representation of the bonds. In this review, we have, therefore, provided some discussion of three-dimensional models of nanoleakage expressions and the process underlying the development of nanoleakage through aging by analyzing existing data for the interfacial analysis of resin-dentin bonds.

\section{Test Methods of Nanoleakage Evaluations}

\section{Microtensile Bond Test and Fractography}

Numerous studies have been conducted based on the microtensile bond test, which was first reported in 1994 [23]. A variety of different specimen shapes can be evaluated using a microtensile bond test, including hourglass-, bar- (or beam-), or cylinder-shaped specimens. A description of the process involved in the preparation of bar-shaped specimens is provided below. Briefly, resin composite-dentin bonded specimens are sectioned using an IsoMet saw to give barshaped specimens with an adhesive area of approximately $1 \mathrm{~mm}^{2}$. The resulting bar-shaped specimens are then attached to a testing apparatus and subjected to a tensile load using a material tester at a crosshead speed of 0.5 to $1.0 \mathrm{~mm} / \mathrm{min}$. After this bond test process, the fractured surfaces of the specimens are sputter coated with gold and observed by SEM.

\section{Nanoleakage Observation by TEM}

A new method was reported in 1995 for the preparation of resin-dentin specimens to allow for the evaluation of their nanoleakage structure by TEM, and this method has subsequently been used in a large number of studies [21, 22]. Briefly, thick resin-dentin bonded disks are prepared and sectioned using an IsoMet saw to make resin-dentin bonded specimens with dimensions of $2 \times 1 \times 5 \mathrm{~mm}$. These specimens are then immersed in an ammoniacal silver nitrate solution for $24 \mathrm{~h}$ and then light cured to allow for the deposition of the silver. After silver staining, the specimens are then processed for TEM examination (non-demineralized specimens).

\section{Nanoleakage Created by Various Adhesives}

\section{Total-Etch Adhesives}

Resin-dentin bonds can be formed using a total-etch (etchand-rinse) or self-etch approach. The total-etch adhesives are subjected to a pretreatment process with acid to allow for the demineralization of the superficial dentin, with the subsequent diffusion of resin monomers of primers or bonding resins, leading to the formation of a hybrid layer within the collagen meshwork. Perfect adhesions can be achieved in this way when the resin becomes embedded in the exposed collagen fibrils without creating empty spaces within the bonds $[1,5$, 11]. However, the formation of empty zones in the bonds would provide a pathway for the penetration of water, which would lead to the degradation of the bonds by bacterial enzymes. The bonds would also be degraded by MMPs, which would be slowly released from the dentin matrix over an extended period $[1,5,11,15,16,17 \bullet, 18 \bullet \bullet, 19]$.

The results of a previous study showed that there was a discrepancy between the thickness of the demineralized collagen layer and the extent to which the resin within the adhesive interface was infiltrated [5]. This zone was found at the fractured surfaces of total-etch adhesive systems after they had been subjected to the microtensile bond test. The authors of this study also speculated that this naked collagen layer had been degraded by hydrolysis, which resulted in the observed long-term reduction in the bond strength. However, there are, currently, no mechanisms available to rationalize the digestion of the collagen fibrils within the bonds. Moreover, it is generally believed that these empty zones are formed at the interface between the hybrid layer and the non-demineralized dentin as layered structures (i.e., microleakage).

In 1994, Sano et al. reported for the first time the detection of a nanoleakage zone within the hybrid layer using silver nitrate staining with subsequent observation by TEM $[16$, 17॰]. The TEM results of this study revealed that there were nanometer-sized porosities in the hybrid layer rather than the restricted area between the hybrid layer and nondemineralized dentin. The silver nitrate sporadically stained the entire hybrid layer. Microleakage is the term used to describe the formation of a gap between the resin and the dentin, where the main reason for the formation of the gap is the polymerization shrinkage of the resin. In contrast, nanoleakage is a term that describes the diffusion of resin molecules within the hybrid layer in the absence of gap formation. Nanoleakage is much less extensive than microleakage. Using this new test method based on a silver tracer, we can easily and clearly detect nanoleakage zones within the bond interfaces by both SEM and TEM.

\section{Self-Etching Adhesives}

Nanoleakage was originally thought to be isolated and unconnected silver grains within the bonds. However, interconnecting, dendritic silver deposits were subsequently observed as tree-shaped structures within the adhesive layers. Moreover, the silver staining of these structures revealed that the hybrid layer and adhesive resin layers existed as polymeric 
matrices, where the water was trapped, resulting in regions of incomplete polymerization and/or hydrogel formation [24]. Strong images showing nanoleakage structures were obtained for the gaps or voids in the hybrid layers resulting from the discrepancies in the acid etching and resin penetration. In contrast, theoretical consideration suggested that no nanoleakage structures would form in the self-etching adhesives. Unfortunately, however, nanoleakage was also found in the resindentin bonds formed with self-etching adhesives [25].

The formation of nanoleakage structures is not only caused by a discrepancy between the depth of demineralization and resin infiltration but can also be caused by the incomplete polymerization or hydrogel formation of the resin. Nanoleakage structures can be classified into a variety of different shapes, including spot mode, reticular mode, and dendritic shapes (water tree) (Fig. 1d). Tay et al. were the first group to propose the two modes of nanoleakage expression (i.e., spot and reticular mode) using single-step adhesives [26]. They also went to speculate that these regions were created by the incomplete removal of water or the interaction of the basic diamine silver ion salts with acidic/hydrophilic resin components. The silver deposits of dendritic shapes were found in polyalkenoic acid copolymer components and were absent from the globular electron-lucent resin phase separations within the droplets [27].

In 2003, Tay and Pashley reported for the first time the concept of a "water tree" as a silver nitrate staining structure within the resin-dentin bonds [23]. There also provided micromorphological evidence of the interconnecting structures between the nanoleakage zones in the hybrid or adhesives layer. In fact, if there were no connections between the nanoleakage structures of each spot or reticular mode, then it would not be possible to observe the nanoleakage structures because the silver nitrate would not be able to penetrate the
Fig. 1 SEM and TEM images or water tree models. a, b SEM images of the fractured surfaces of resin-dentin bonds that had been created using a one-bottle selfetching adhesive. $\mathbf{c}$ TEM images of a resin-dentin interface that had been created using a onebottle self-etching adhesive. The specimens were stored in water for 8 years prior to being analyzed by TEM. d Schematic illustration of the aging patterns of nanoleakage. The black arrows indicate the spot-mode structure of the nanoleakage expression, which consisted of isolated spots of silver grains that were dispersed upon the leakage layer (C). e Three-dimensional tree model. The morphological characteristics of each region of the water tree (left figure). This tree is a model of the water tree structures generally formed at the resin-dentin interface. This tree was sectioned in one direction, and the resulting sectioned end of tree branch is shown in the two figures to the right. These situations were simulated by specimen preparation with subsequent observation by TEM. $L$ leakage layer, $B$ bonding resin, $C$ composite resin. The sectioned end of tree branch has been colored in yellow (the two figures to the right)
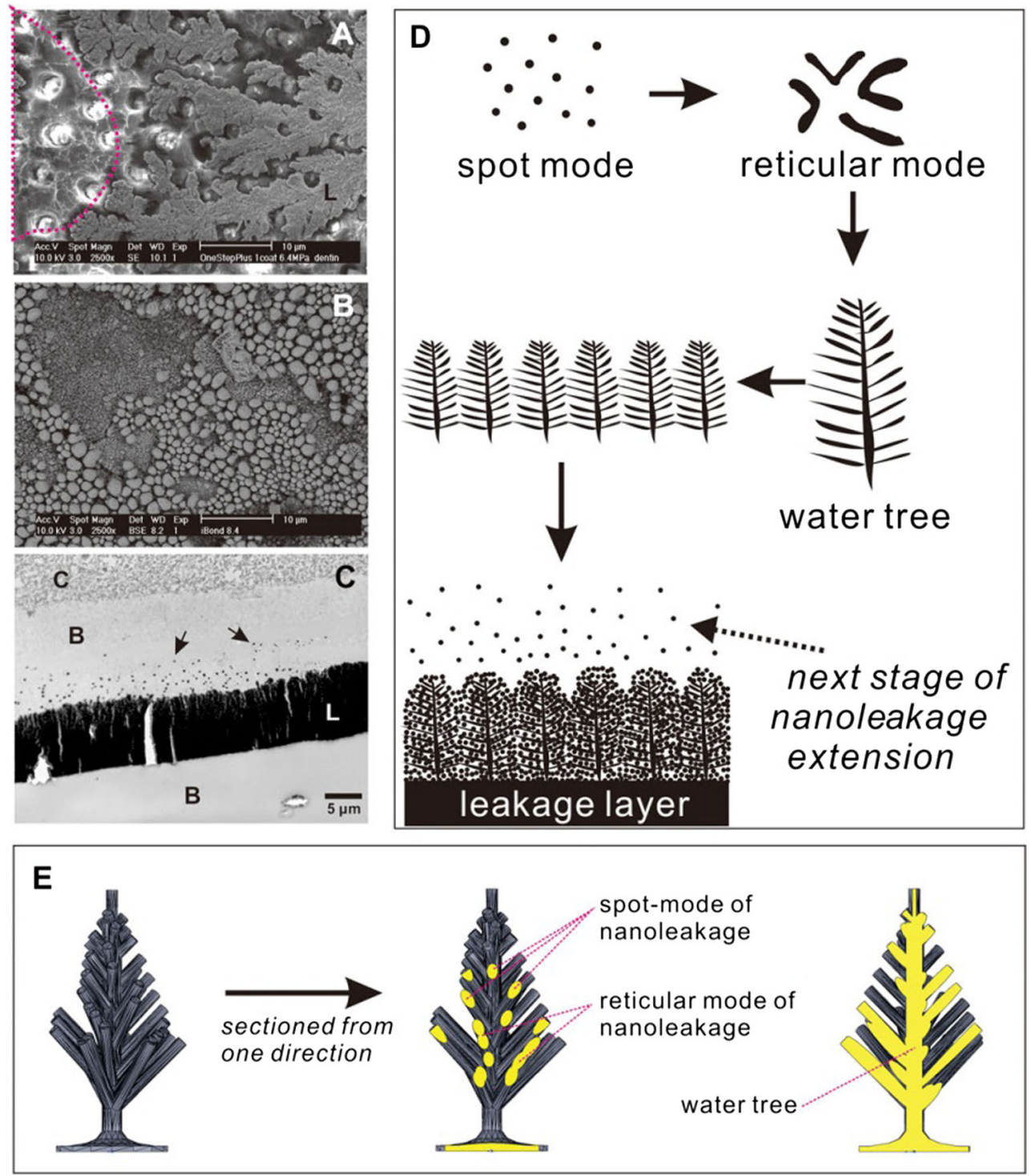
resin. The silver nitrate can infiltrate the adhesive interface by directly attacking the interface or moving into the dentinal tubules during the preparation of the specimens. Additional routes or pathway are also required for the penetration of silver into the hybrid layer or bonding resin.

We measured the outward movement of the water (i.e., from the dentin to the bonding resin) within the resin-dentin bonds using an instrument, which was specially designed to measure the movement of water [28]. This device allowed us to record diffusion-induced water movements during the placement or light curing of adhesive resins, and the results suggested that the movement of water could contribute to creation of water-filled channels or voids within the bonds [23]. The water itself was supplied from the dentinal tubules. Notably, these water movements became larger when hydrophilic adhesives such as all-in-one adhesives were used in the study (unpublished results). In addition, the cured bonding resin was shown to be nothing more than permeable membrane [29]. Water movements through the cured resin films were recorded by osmosis, which was induced by a $\mathrm{CaCl}_{2}$ or HEMA solution. The extent of this water movement was found to be greater for hydrophilic adhesive, compared with their hydrophobic counterpart. Furthermore, the movement or entrapment of water within the adhesive resin led to a decrease in the shrinkage of the resin during the polymerization bonding process $[30,31]$. These in situ test results also provided evidence of water sorption to the bonding resin during the bonding process. In all these experiments, the resin-dentin bonds were generally characterized after $24 \mathrm{~h}$ of bonding. The results of these two in situ studies provide clear evidence in support of the formation of nanoleakage structures in the bonds over a short time interval (i.e., $30 \mathrm{~min}$ ) at the start of the bonding procedure [28-31]. It is therefore likely that water sorption is one of the main reasons for the formation of nanoleakage structures within the resin-dentin bonds. In terms of the morphological characteristics of these bonds, the SEM images of a replica of vital acid-etched dentin showed the transudation of dentinal fluid to the dentin surface [32]. This process was followed by the formation of water blisters at the resin-dentin interface, with the water molecules coming from the dentinal fluid trapped by the water-immiscible resins. Water blisters of this type can be found in hydrophilic adhesives by dentinal surface observation by SEM (three-dimensional approach) and interfacial observations by TEM (two-dimensional approach).

\section{Nanoleakage and Bond Degradation}

\section{Two-Dimensional Evaluation}

The degradation of resin-dentin bonds has been examined over an extended time period by long-term water storage testing. The bonding strength of many resin adhesives decreases following their long-term (6 months or 1 year) storage in water during in vitro or long-term in vivo tests [33-36]. The extent of nanoleakage expression also increases in these after long-term storage in water $[11,37]$. Both of these issues can be attributed to the penetration of water into the adhesive interface via nanoleakage pathways, followed by the slow hydrolysis of the resin polymer, which would lead to further increases in the volume of the nanoleakage [11]. This degradation mechanism must therefore be caused by the hydrolysis degradation of the resin because there were no increases in the formation of nanoleakage structures or decrease in the bonding strength when the bonded specimens were immersed in mineral oil $[13,15]$. Moreover, the direct exposure of the adhesive interface to water (using the bar-shaped resin-dentin bonded specimens) leads to an increase in the rate of degradation, compared with the resin-dentin bulk specimens [2, 21].

When pulpal pressure was applied to the bonded interface, there was a significant increase in the rate of the reduction in the bond strength, which suggested that the movement of dentinal fluid in bonded interface could accelerate the degradation of the bonds [15]. Figure 1a shows the fractured surface of the resin-dentin bond with significant silver nitrate penetration shown in the right part of the figure. The silver nitrate penetration continued, with the morphological characteristics suggesting the formation a layer. However, the left part of the image shows that there was a hybrid layer with some dentinal tubules. Careful consideration of the images revealed the presence of nanoleakage with a spot-mode structure in the hybrid layer (Fig. 1a). Figure 1b shows that the balloon-shaped silver deposits had formed at the adhesive interface on the fractured surface and that these deposits could be localized from the hybrid layer to the bonding resin layer or their border. Silver deposits of this type can weaken the bonding performance of the resin, which would result in the formation of fracture in this region after the bond test. Figure 1c shows the aged bonded interface created using one-bottle self-etching adhesives following their storage in water for 8 years. The silver nitrate layer was observed between the adhesive resin and the adhesive resin interface.

During the bonding procedure, the bonding resins are applied to the dentin surface using air-blowing and light-curing techniques. Additional bonding resin can then be applied to the cured adhesive layer (consecutive coatings). In this process, the first of the adhesives to be applied to the dentin surface forms a thick unpolymerized resin layer on top of the well-cured adhesive layers [38-40]. This type of nanoleakage is occasionally found in one-bottle self-etching adhesives because they contain significant quantities of water, ethanol, and acetone as solvents. These hydrophilic adhesives can therefore hamper the polymerization of the outer resin surface by introducing oxygen. The propagation of the 
nanoleakage may consequently start at these legions during the water storage. Furthermore, numerous spot-mode nanoleakage structures were observed on the leakage layer (Fig. 1c, black arrows). This result therefore represents first example of a nanoleakage propagation process for the next stage of bond degradation.

\section{Three-Dimensional Evaluation}

We recently developed a model of nanoleakage propagation in resin-dentin bonds to evaluate the changes in these bonds during aging (Fig. 1d). The results of this model were as follows. At the initial nanoleakage stage, we could see the nanoleakage existed as a simple spot. After aging, the spotmode structures of individual nanoleakage connected with each other to form a reticular-mode nanoleakage structure. During the subsequent degradation stage, more spot-mode and reticular-mode nanoleakage structures joined together to become dendritic shape or water tree. Many water tree formations were generated at the adhesive interface with the structures occurring simultaneously.

Figure 1e shows the three-dimensional images of the tree models (http://www.thigiverse.com/), which were sectioned at one quarter of the model from an anterior perspective using CAD software (SolidWorks Simulation 2011; SolidWorks Corporation, MA, USA). The sectioned areas shown in the figure (sectioned at the end of the tree branch) have been colored in yellow. We simulated the three-dimensional tree model as a water tree. The sectioning of the water tree structure at the resin-dentin interface of each specimen was conducted using a diamond knife for the preparation of the TEM samples. Three simulated water tree structures were prepared at the adhesive interface. The structure shown at the far left of the figure is the uncut tree. When we cut the tree once in one direction, we could clearly see the spot- and reticular-shaped ends of the tree branches (colored yellow in the center figure). When we cut the tree down the center, we could clearly see the dendritic-shaped end (colored yellow in the right figure). These results suggested that, even if there were many water tree structures at the bonded interface, we would invariably observe the structures as spot-mode or reticular-mode nanoleakage structures. Dendritic-mode structures would be observed much less frequently because the resulting TEM images would only provide two-dimensional information. It is important to note that the images revealed that the spotmode structures of the nanoleakage were all connected to each other by small water pathway that were not observed by highresolution TEM. With this in mind, the morphological phases of the structures can be seen as water bush or leakage layers because they were connected laterally at the bonded interface. When we observed this type of leakage from the upper part of the bonded structure by SEM, we could see a large number of balloon-shaped nanoleakage or band layer structures similar to those shown in Fig. 1a, b, respectively. These structures ultimately became band layer like, with the nanoleakage propagation process starting from these layers as spot-mode structures (Fig. 1c). During this process, significant amounts of water would be supplied from the leakage layer for the formation of additional nanoleakage structures. We believe that the leakage layer represents the most severe form of nanoleakage structure and that it is almost equal to that of a microleakage. However, we were unable to find this type of shape by TEM because leakage zones of this type can be readily broken down by the procedures used for the preparation of specimens of $100 \mathrm{~nm}$ in thickness during TEM sectioning. This type of degradation is typically found at the adhesive interfaces of hydrophilic one-bottle self-etching adhesives [41]. After the bar-shaped specimens had been stored in water for 1 year, we conducted fractography at the dentin side of the specimens [2]. The resin materials in the hybrid layer had been gradually extracted from the periphery to the central portion of the resin-dentin bonded beams. These results suggested that the degradation of the bond had occurred as a consequence of the storage water coming into direct contact with the adhesive interface. Similar results were also obtained following the silver nitrate staining of the aged specimens [41]. In this case, we had made the resin-dentin bonded specimens with hydrophilic one-bottle self-etching adhesives. After 1 year of water storage, we conducted silver nitrate staining followed by SEM examination. Observation of the interfacial area by SEM revealed the presence of silver deposits at the interface between the adhesive and resin composite. The unpolymerized layer on the upper portion of the bonding resin readily formed as an imperfect binding zone, which subsequently formed a nanoleakage structure that propagated in the presence of water. This degradation pattern has never before been observed in hydrophobic adhesives such as two-step self-etching systems or two-/three-step total-etching adhesives. Although imperfect bonding can occur as a consequence of numerous factors, we can observe all of the different nanoleakage patterns after only $24 \mathrm{~h}$ of bonding. During the aging process, the number of water tree or leakage zones at the adhesive interface can increase considerably. However, more detailed three-dimensional analyses are required to develop a better understanding of this process [42]. Further work towards the combination of two-dimensional TEM analysis with three-dimensional SEM analysis [43] or the development of new special devices capable of three-dimensional analysis would also be useful [44].

\section{Summary}

The use of silver nitrate represents an effective tracer method for observing the micromorphological structure of the resindentin adhesive interface by TEM. Analytical examinations of 
this type allow for the structure of the bonding interface to be considered in greater detail, especially any structural defects results from the adding effects. However, it is important to note that the images acquired by TEM should be given careful consideration because they are only two dimensional. Further study is therefore required to evaluate the micromorphological characteristics of resin-dentin bond structures using threedimensional approaches.

\section{Compliance with Ethics Guidelines}

Funding This work was supported in part by Grants-in-Aid for Scientific Research (Nos. 26462953 and 26293409) from the Japan Society for the Promotion of Science (JSPS). Some of the TEM experiments described in this review were performed at the Research Center for UltraHigh Voltage Electron Microscopy of Osaka University. The authors are grateful to Dr. Ryusuke Kuwahara and Dr. Yoshinori Muranaka for their technical support.

Conflict of Interest Masanori Hashimoto, Satoshi Yamaguchi, and Imazato Satoshi declare that they have no conflicts of interest.

Informed Consent Human premolars were collected from patients after their informed consent had been obtained under a protocol reviewed and approved by the Human Assurance Committee of Osaka University (H25-E23).

\section{References}

Papers of particular interest, published recently, have been highlighted as:

- Of importance

.. Of major importance

1. Hashimoto $\mathrm{M}$ et al. In vivo degradation of resin-dentin bonds in humans over 1 to 3 years. J Dent Res. 2000;79(6):1385-91.

2. Hashimoto $\mathrm{M}$ et al. Micromorphological changes in resin-dentin bonds after 1 year of water storage. J Biomed Mater Res. 2002;63(3):306-11.

3. Sano $\mathrm{H}$ et al. Long-term durability of dentin bonds made with a self-etching primer, in vivo. J Dent Res. 1999;78(4):906-11.

4. De Munck J et al. Four-year water degradation of a resin-modified glass-ionomer adhesive bonded to dentin. Eur J Oral Sci. 2004;112(1):73-83.

5. Nakabayashi $\mathrm{N}$ et al. A tensile test to facilitate identification of defects in dentine bonded specimens. J Dent. 1998;26(4):379-85.

6. Anchieta RB et al. Effect of partially demineralized dentin beneath the hybrid layer on dentin-adhesive interface micromechanics. J Biomech. 2015;48(4):701-7.

7. Hashimoto $\mathrm{M}$ et al. Permeability of adhesive resin films. J Biomed Mater Res. 2005;74(2):699-705.

8. Ito $\mathrm{S}$ et al. Effects of resin hydrophilicity on water sorption and changes in modulus of elasticity. Biomaterials. 2005;26(33): 6449-59.

9. Walter R et al. One-year water sorption and solubility of "all-inone" adhesives. Braz Dent J. 2013;24(4):344-8.

10. Hashimoto $\mathrm{M}$ et al. Measurements of volatile compound contents in resins using a moisture analyzer. Eur J Oral Sci. 2010;118(1):94-9.
11. Hiraishi $\mathrm{N}$ et al. Water concentration in self-etching primers affects their aggressiveness and bonding efficacy to dentin. J Dent Res. 2005;84(7):653-8.

12. Marghalani HY et al. Sorption and solubility characteristics of selfadhesive resin cements. Dent Mater. 2012;28(10):187-98.

13. Tay FR et al. Aging affects two modes of nanoleakage expression in bonded dentin. J Dent Res. 2003;82(7):537-41.

14. Ito $\mathrm{S}$ et al. Water sorption/solubility of self-etching dentin bonding agents. Dent Mater. 2010;26(7):617-26.

15. Feitosa VP et al. Hydrolytic degradation of the resin-dentine interface induced by the simulated pulpal pressure, direct and indirect water ageing. J Dent. 2012;40(12):1134-43.

16. De Munck J et al. Four-year water degradation of total-etch adhesives bonded to dentin. J Dent Res. 2003;82(2):136-40.

17. Pashley DH et al. Collagen degradation by host-derived enzymes during aging. J Dent Res. 2004;83(3):216-21. This manuscript represents the first study of the collagen degradation of hostderived enzymes.

18.• Bertassoni LE et al. The dentin organic matrix-limitations of restorative dentistry hidden on the nanometer scale. Acta Biomater. 2012;8(7):241-33. This review article represents the interaction of resin to dentinal collagen by the basic theory.

19. Liu $\mathrm{Y}$ et al. Limitations in bonding to dentin and experimental strategies to prevent bond degradation. J Dent Res. 2011;90(8): 953-68.

20. Mazzoni A et al. Role of dentin MMPs in caries progression and bond stability. J Dent Res. 2015;94(2):241-51.

21. Sano $\mathrm{H}$ et al. Nanoleakage: leakage within the hybrid layer. Oper Dent. 1995;20(1):18-25.

22. Sano $\mathrm{H}$ et al. Comparative SEM and TEM observations of nanoleakage within the hybrid layer. Oper Dent. 1995;20(4):160-7.

23. Sano $\mathrm{H}$ et al. Relationship between surface area for adhesion and tensile bond strength-evaluation of a micro-tensile bond test. Dent Mater. 1994;10(4):236-40.

24. Tay FR et al. Water treeing - a potential mechanism for degradation of dentin adhesives. Am J Dent. 2003;16(1):6-12.

25. Tay FR et al. How can nanoleakage occur in self-etching adhesive systems that demineralize and infiltrate simultaneously? J Adhes Dent. 2002;4(4):255-69.

26. Tay FR et al. Two modes of nanoleakage expression in single-step adhesives. J Dent Res. 2002;81(7):472-6.

27. Kelli KL et al. Submicron hiati in acid-etched dentin are artifacts of desiccation. Dent Mater. 2003;19(1):60-8.

28. Hashimoto $\mathrm{M}$ et al. Diffusion-induced water movement within resin-dentin bonds during bonding. J Biomed Mater Res B Appl Biomater. 2006;79(2):453-8.

29. Hashimoto $\mathrm{M}$ et al. Permeability of adhesive resin films. J Biomed Mater Res B Appl Biomater. 2005;74(2):699-705.

30. Hashimoto $\mathrm{M}$ et al. Contraction stress in dentin adhesives bonded to dentin. J Dent Res. 2006;85(8):728-32.

31. Hashimoto $\mathrm{M}$ et al. Polymerization contraction stress in dentin adhesives bonded to dentin and enamel. Dent Mater. 2008;24(10): 1304-10.

32. Tay FR et al. Have dentin adhesives become too hydrophilic? J Can Dent Assoc. 2003;69(11):726-31.

33. Walter R et al. Two-year bond strengths of "all-in-one" adhesives to dentine. J Dent. 2012;40(7):549-55.

34. Garcia-Godoy F et al. Long term degradation of enamel and dentin bonds: 6-year results in vitro vs. in vivo. Dent Mater. 2010;26(11): $1113-8$.

35. Abdalla AL et al. Effect of long-term water aging on microtensile bond strength of self-etch adhesives to dentin. Am J Dent. 2010;23(1):29-33.

36. Hashimoto $\mathrm{M}$ et al. A review-micromorphological evidence of degradation in resin-dentin bonds and potential preventional solutions. J Biomed Mater Res. 2010;92(1):268-80. 
37. Kim YK et al. Biomimetic remineralization as a progressive dehydration mechanism of collagen matrices - implications in the aging of resin-dentin bonds. Acta Biomater. 2010;6(9):3729-39.

38. Navarra CO et al. Degree of conversion of two-step etch-and-rinse adhesives: in situ micro-Raman analysis. J Dent. 2012;40(9):711-7.

39. Shawkat ES et al. Oxygen inhibition and incremental layer bond strengths of resin composites. Dent Master. 2009;25(11):1338-46.

40. Gauthier MA et al. Oxygen inhibition in dental resins. J Dent Res. 2005;84(8):725-9.
41. Hashimoto $\mathrm{M}$ et al. In vitro durability of one-bottle resin adhesives bonded to dentin. Dent Mater J. 2007;26(5):677-86.

42. Coutinho $\mathrm{E}$ et al. Nanoleakage distribution at adhesive-dentin interface in 3D. J Dent Res. 2011;90(8):1019-25.

43. Suppa $P$ et al. Nanoleakage within the hybrid layer: a correlative FEISEM/TEM investigation. J Biomed Mater Res. 2005;73(1):714.

44. Neves A et al. 3D-microleakage assessment of adhesive interfaces: exploratory findings by $\mu \mathrm{CT}$. Dent Mater. 2014;30(8):799-807. 